\title{
GERMINACIÓN Y ESTABLECIMIENTO DE PLÁNTULAS DE Pterocereus GaUmerl, unA CACTÁCEA COLUMNAR, RARA Y ENDÉMICA DE YUCATÁN, MÉXICO
}

\author{
Martha Méndez'1, Alfredo Dorantes, Gabriel Dzib, Jorge Argáez y Rafael Durán \\ Centro de Investigación Científica de Yucatán, A.C. (CICY), Calle 43 No. 130, \\ Col. Chuburná de Hidalgo, C.P. 97200 Mérida, Yucatán, México. \\ ${ }^{1}$ Autor para la correspondencia. Correo-e: mar@cicy.mx
}

\begin{abstract}
Resumen: Se evaluó el nodricismo y la depredación sobre la germinación y el establecimiento de nuevos individuos de Pterocereus gaumeri. Se realizó un experimento de campo con diseño factorial, considerando el factor asociación con cinco niveles (cuatro nodrizas y claros), el factor depredación con dos (protegidas y expuestas) y el factor orientación con cuatro (cuatro puntos cardinales). No hubo diferencias significativas en la germinación entre claros y bajo las nodrizas, ni entre especies, pero sí entre semillas protegidas de la depredación y las expuestas. Para el factor orientación no se encontraron diferencias en ningún caso. La supervivencia de las plántulas bajo nodrizas fue significativamente mayor que en los claros y se presentó una gran depredación de plántulas. Estos resultados sugieren que el reclutamiento de nuevos individuos a las poblaciones de $P$. gaumeri es una fase crítica de su ciclo de vida y contribuyen a explicar la rareza de esta cactácea amenazada.
\end{abstract}

Palabras clave: cactácea rara, germinación, nodricismo, supervivencia de plántulas.

\begin{abstract}
We examined the facilitative effect of nurse plants and the negative effect of predation on the germination and establishment of new individuals of Pterocereus gaumeri. The field experiment followed a factorial design, with five levels of association (four nurse plants and open space), four levels of orientation (North, South, East, and West) and two levels of predation (exclusion and non-exclusion of predators). There were no statistical differences between germination rates in open sites and under nurse plants, neither between nurse plants; however, seed germination was higher in the protected treatments. There were no statistical differences in the orientation factor for germination and survival. Seedling survival was higher under nurse plants than in open sites and seedling predation was very high. Our results showed that the recruitment of new individuals of $P$. gaumeri is a critical stage of the life cycle of the species and help explain the degree of rarity of this threatened cactus species.

Key words: germination, nurse plants, rare cacti, seedlings survivorship.
\end{abstract}

$\mathbf{E}_{\mathrm{d}}^{1}$ conocimiento de los factores y procesos que afectan y determinan la germinación de las semillas y el establecimiento de plántulas es fundamental para el entendimiento de la dinámica de las poblaciones vegetales. En el caso de las poblaciones de cactáceas, las cuales se distribuyen tanto en ambientes desérticos, áridos y semiáridos, como matorrales xerófilos y selvas bajas tropicales, los individuos en las primeras fases de su ciclo de vida se ven expuestos a condiciones ambientales extremas (sequías severas, suelos con altas temperaturas y bajo contenido de humedad), así como a las altas tasas de depredación (Valiente-Banuet y Ezcurra, 1991; Godínez-Álvarez et al., 1999; Ruedas et al., 2000). Por ello, los desiertos han sido modelo de estudio para el entendimiento de las interacciones entre especies vegetales, en particular de aquellas asociaciones que determinan que las plántulas de algunas especies se establezcan bajo las copas de los individuos de otras especies.

En términos generales, se reconoce que los árboles y los arbustos modifican el microambiente debajo de su copa, facilitando el establecimiento de plántulas de especies, tanto anuales como perennes. A este tipo de interacción se le conoce comúnmente como nodricismo. Al parecer, este tipo de asociación es fundamental en la germinación y el reclutamiento de nuevos individuos en las poblaciones de algunas especies de cactáceas (Steenbergh y Lowe, 1969; Franco y Nobel, 1989; Valiente-Banuet y Ezcurra, 1991; Suzán et al., 1996; Nolasco et al., 1997; Mandujano et al., 1998; Contreras y Valverde, 2002; Esparza-Olguín et al., 2002; Godínez-Álvarez et al., 2003). Las plantas nodriza 
modifican los factores que tienen impacto en el balance hídrico de los organismos y en la disponibilidad del agua en el suelo, ya que alejadas de su copa, las plántulas están sometidas a condiciones extremas de temperatura y radiación (Shreve, 1931; McAuliffe, 1988; Fowler, 1986; Valiente-Banuet et al., 1991a).

Se han propuesto diversas explicaciones para este fenómeno, como el que las plantas nodriza proporcionan protección contra la radiación y la pérdida excesiva de agua (Shreve, 1931; Turner et al., 1966; Nobel, 1980), disminuyen la temperatura del suelo (Valiente-Banuet et al., 1991a; Arriaga et al., 1993), brindan protección a semillas y plántulas contra la depredación y contra posibles daños mecánicos (Niering et al., 1963; Steenbergh y Lowe, 1977; McAuliffe, 1984; Valiente-Banuet y Ezcurra, 1991). También se ha propuesto que el incremento en la concentración de nitrógeno del suelo, bajo la copa de las plantas nodriza, facilitaría el establecimiento de plántulas (GarcíaMoya y McKell, 1970). Sin embargo, se ha encontrado que en algunos casos la fertilidad de los suelos es menor debajo de la copa de las nodrizas que en los espacios abiertos (Valiente-Banuet et al., 1991b).

El fenómeno de nodricismo ha sido reportado para todas las formas de vida de la familia cactácea (Steenbergh y Lowe, 1969; Franco y Nobel, 1989; Valiente-Banuet y Ezcurra, 1991; Arriaga et al., 1993; Suzán et al., 1996; Mandujano et al., 1998; Rodríguez y Ezcurra, 2000; Zuñiga et al., 2005). En particular, para las cactáceas columnares se ha documentado esta asociación en Carnegia gigantea (Niering et al., 1963; Turner et al., 1966; Steenbergh y Lowe, 1977, 1983; Nobel, 1980; Vandermeer, 1980; Hutto et al., 1986; Franco y Nobel, 1989), en Cephalocereus hoppenstedtii (Valiente-Banuet et al., 1991a), en Neobuxbaumia tetetzo (Valiente-Banuet et al., 1991a, b), en Stenocereus thurberi (Arriaga et al., 1993) y en Pachycereus hollianus (Godínez-Álvarez y Valiente-Banuet, 1998).

La mayor parte de estos trabajos han sido desarrollados en zonas desérticas de diferentes partes del mundo. Aunque la selva baja caducifolia de Yucatán no presenta condiciones tan extremas como los desiertos, el período de sequía es muy prolongado, abarcando hasta siete meses del año. Esto hace que alrededor de $95 \%$ de los árboles y arbustos pierdan su follaje durante la época de sequía, creando condiciones extremas de insolación y temperatura a nivel del piso de la selva. En este contexto, nuestro estudio pretende explorar los mecanismos que limitan el reclutamiento de nuevos individuos en las poblaciones una cactácea columnar: Pterocereus gaumeri.

Pterocereus gaumeri (Britton et Rose) T.MacDougall et Miranda es una especie endémica de la Península de Yucatán, cuya distribución se restringe básicamente a la selva baja con cactáceas candelabriformes del norte de Yucatán. Es considerada una especie sujeta a protección especial (Pr) en la NOM-059-2001 y está clasificada como especie en peligro por la IUCN (Walter y Gillett, 1998). Sus poblaciones presentan bajas densidades (alrededor de 100 adultos/ha), en comparación con otras cactáceas columnares que comparten el mismo hábitat (Stenocereus eichlamii: 400 adultos/ha; Pilosocereus gaumeri: 165 adultos/ha; Méndez et al., 2004), así como de cactáceas columnares de otras áreas (Neobuxbaumia tetetzo: 1,5002,000 plantas/ha en el Valle de Tehuacán; Valiente-Banuet y Ezcurra, 1991).

Por lo limitado de su distribución y las bajas densidades poblacionales que presenta, es considerada como una especie rara de acuerdo con Reveal (1981), quien define a la rareza como el estado actual de un taxón, el cual por cualquier combinación de factores físicos o biológicos, se encuentra restringido en sus números, en el área que ocupa, o en ambos, a un nivel que es demostrablemente menor que otros organismos de entidades taxonómicas comparables. El estudio de las especies raras es particularmente importante debido al riesgo de extinción que tienen estas especies (Fielder y Jain, 1992; Harrison, 1993), ya que son muy vulnerables a la perturbación y destrucción de los ambientes en que habitan, situación que se agrava día con día.

Se ha documentado que de las fases del ciclo de vida de $P$. gaumeri, la germinación y el establecimiento de plántulas son periodos críticos en la dinámica de las poblaciones de esta especie (Méndez et al., 2004), que además de presentar bajas densidades poblacionales, se encuentra sujeta a otras presiones, debido al fraccionamiento de su hábitat como producto de diversas actividades antropogénicas.

Considerando que el fenómeno del nodricismo ha sido reportado para otras especies de cactáceas columnares, se pretende evaluar el efecto de este fenómeno en la germinación y el establecimiento de nuevos individuos de $P$. gaumeri. Adicionalmente, se explorará si existe relación entre la germinación y la supervivencia de plántulas y su orientación con respecto a la planta nodriza. Asimismo, se pretende analizar el efecto de la depredación de semillas y de plántulas en el reclutamiento de los nuevos individuos.

\section{Materiales y métodos}

La especie. Pterocereus gaumeri es una cactácea columnar que puede alcanzar hasta $8 \mathrm{~m}$ de altura, con tallos que se ramifican después de alcanzar los $2 \mathrm{~m}$ de altura (para una descripción completa de la especie ver Bravo-Hollis, 1978).

Las flores son de color crema claro o verde amarillento. Sólo se produce una flor por areola. El período de floración inicia al principio de enero, aunque unos pocos individuos pueden empezar a producir botones a finales del mes de diciembre. La floración concluye en mayo, con un pico de floración en marzo. La antesis es nocturna y para cada individuo solamente abren de una a tres flores por noche; las 
flores son polinizadas por murciélagos $\mathrm{y}$, aunque permanecen abiertas hasta la mañana siguiente, los insectos diurnos no son polinizadores efectivos (Méndez et al., 2005).

El período de producción de frutos maduros se extiende desde la segunda quincena de marzo hasta finales de junio, presentando un máximo durante la primera mitad del mes de mayo. Hay una gran pérdida de frutos inmaduros debido fundamentalmente a la depredación (Méndez et al., 2005).

Área de estudio. Pterocereus gaumeri se presenta principalmente en la selva baja caducifolia con cactáceas candelabriformes (Miranda y Hernández-X., 1964; Olmsted et al., 1999), la cual está ubicada en una franja de 10 a $15 \mathrm{~km}$ de ancho que se extiende desde el sur del puerto de Sisal hasta el sur de Ría Lagartos, Yucatán. Esta selva se caracteriza por la presencia de árboles de 8 a $10 \mathrm{~m}$ de altura, los cuales presentan troncos de madera dura, retorcidos y delgados, la mayoría de los cuales tienen diámetros que no exceden de $10 \mathrm{~cm}$; prácticamente $100 \%$ de los árboles tiran su follaje durante la época de sequía. Los elementos más conspicuos en el estrato arbóreo son Acacia gaumeri, Acacia pennatula, Alvaradoa amorphoides, Bursera simaruba, Mimosa bahamensis, Gymnopodium floribundum, Hampea trilobata, Havardia albicans y Metopium brownei. Entre las especies de la familia Cactaceae encontramos a Acanthocereus pentagonus, Stenocereus eichlamii, Nopalea gaumeri, Nopalea inaperta, Mammillaria gaumeri, Opuntia dillenii, Hylocereus undatus y Selenicereus donkelaarii. Cabe destacar que en este tipo de vegetación se presentan numerosas especies endémicas de la Península de Yucatán (Espadas et al., 2003).

El estudio se llevó a cabo en el rancho Chumhabín (21 ${ }^{\circ} 18^{\prime} \mathrm{N}$; 8910' O). Allí, los árboles alcanzan una altura máxima de $8 \mathrm{~m}$ y el diámetro de la mayoría no excede 15 $\mathrm{cm}$. El terreno se encuentra completamente cercado, por lo que se encuentra a salvo del pisoteo por el ganado.

Diseño experimental. En Chumhabín se estableció una parcela de 5,000 $\mathrm{m}^{2}$ y se llevó a cabo una evaluación de la abundancia relativa de las especies de árboles y arbustos adultos presentes en ella. A partir de esta evaluación se seleccionaron dos especies perennifolias (Amyris attenuata Standl. y Guaiacum sanctum L.) y dos caducifolias (Acacia gaumeri S.F.Blake y Malpighia punicifolia L.); en ambos casos fueron las más abundantes en el sitio de estudio. Debido a que gran parte del año los individuos pierden sus hojas por efecto de la sequía, nos interesaba indagar si esto tendría efecto en la supervivencia de las plántulas. A fin de comparar el efecto de las nodrizas con condiciones de mayor exposición a la radiación solar, se seleccionaron claros de aproximadamente $10 \mathrm{~m}$ de diámetro.

Para realizar este estudio se identificaron tres factores que pueden ser relevantes para la germinación y la super- vivencia: el factor asociación, con cinco niveles (cuatro especies de plantas nodriza y claros), el factor depredación, con dos niveles (protección y exposición a la depredación) y el factor orientación, con cuatro niveles (N, S, E y O). El factor orientación se tomó en cuenta debido a que algunos autores han reportado que la inclinación del sol puede ser un factor que influye en el establecimiento de plántulas en las zonas áridas y semiáridas (ValienteBanuet y Ezcurra, 1991).

De acuerdo con el diseño especificado, para cada nodriza y en cada claro se probó la condición de orientación en los cuatro puntos cardinales, así como la protección y exposición a depredadores. La combinación de los tres factores con sus respectivos niveles da lugar a 40 tratamientos, resultado de los dos niveles de protección, los cinco niveles de asociación y las cuatro orientaciones. Cada una de las 40 combinaciones posibles fue replicada cinco veces.

Durante julio de 1998 se recolectaron semillas de 30 frutos de diferentes individuos. Las semillas fueron sembradas de forma superficial en lotes de 30 semillas cada uno (unidades experimentales) en una superficie aproximada de $100 \mathrm{~cm}^{2}$, en la base de los individuos seleccionados como nodrizas o en los claros, teniendo mucho cuidado de no modificar la estructura del suelo. En los claros las unidades experimentales se colocaron en la zona central en dirección a los cuatro puntos cardinales con relación al centro del claro. Para la exclusión de los depredadores las semillas se protegieron con jaulas elaboradas con malla metálica de 4 mm de luz $(15 \times 15 \times 10 \mathrm{~cm})$, las cuales fueron parcialmente enterradas en el suelo para cubrir las semillas destinadas a este tratamiento. Para excluir a las hormigas, se aplicó, cada semana, paratión metílico alrededor de las jaulas.

La siembra se llevó a cabo el 5 de agosto de 1998, durante la época de lluvias. Durante el mes siguiente, cada semana se registró la germinación de las semillas, y posteriormente cada 10 días. El experimento concluyó cuando no se observó incremento en el número de semillas germinadas durante 20 días seguidos. Las últimas semillas germinaron el 6 de octubre del mismo año.

Para evaluar la viabilidad de las semillas, de forma paralela se pusieron a germinar semillas en condiciones de laboratorio. Del mismo lote de semillas que se sembraron en el campo, se tomaron cinco grupos de 30 semillas cada uno, los cuales se sembraron en agrolita y se colocaron en una cámara de germinación, con un régimen de temperatura de $35^{\circ} / 30^{\circ} \mathrm{C}$ y un fotoperíodo de $12 \mathrm{~h}$, regándolas cada tercer día para mantener la humedad del sustrato.

Una vez concluida la germinación de las semillas en el campo, en el mes de octubre se inició el registro de la mortalidad de plántulas y sus posibles causas, lo cual permitió registrar el éxito en su establecimiento, así como la mortalidad que se presentó en cada uno de los tratamientos establecidos. La supervivencia de las plántulas fue evalua- 
da cada semana durante el primer mes de estudio, posteriormente de forma mensual durante el primer año y anualmente durante el período subsiguiente. El período total de estudio abarcó poco más de tres años y la evaluación concluyó el 11 de octubre de 2001.

Las condiciones climáticas prevalecientes para el período de estudio se obtuvieron de los datos de precipitación y temperatura registrados en la estación meteorológica de Telchac Puerto, la más cercana al sitio de estudio.

Análisis de datos. Las diferencias en la proporción (p) de semillas germinadas en cada factor se analizaron mediante un análisis de varianza; para normalizar los datos, se utilizó la transformación arcoseno ( $\left.\mathrm{p}^{\prime}=\operatorname{arcsen} \sqrt{ } \mathrm{p}\right)$, de acuerdo con Zar (1984).

Con el fin de analizar el establecimiento de las plántulas se elaboraron curvas de supervivencia $\left(\log n_{x}\right)$ para cada uno de los factores considerados; las curvas obtenidas se compararon mediante el análisis de rangos logarítmicos de Peto y Peto (Pyke y Thompson, 1986).

Para evaluar el efecto de la asociación en la supervivencia de plántulas se compararon las curvas obtenidas en los cinco niveles del factor asociación (cuatro especies de nodriza y claros), incluyendo a las plantas protegidas y expuestas a la depredación; posteriormente se comparó la supervivencia obtenida en cada especie con la de los claros. Con el fin de explorar si se presentó una supervivencia diferencial de las plántulas como producto de las características de las especies nodrizas, se compararon las curvas de supervivencia obtenidas para las diferentes especies seleccionadas.

El efecto de la orientación en la probabilidad de supervivencia de las plántulas se obtuvo tomando en cuenta la supervivencia de las plantas en cada punto cardinal, incluyendo a las plantas protegidas y expuestas a la depredación que se encontraban creciendo en los claros y bajo las nodrizas. Para evaluar el efecto de la depredación en la supervivencia de las plántulas se compararon las curvas de supervivencia obtenidas para los individuos protegidos de la depredación y la de los expuestos a los depredadores.

A fin de complementar el análisis anterior e integrar la varianza observada en las variables de respuesta, se efectuó un análisis de varianza factorial que nos permitiera dilucidar el efecto de los factores y de las interacciones entre factores.

\section{Resultados}

Germinación. Los porcentajes promedio de germinación obtenidos en el experimento con las semillas protegidas de la depredación oscilaron entre 18.7 y $44.7 \%$. En las semillas expuestas a la depredación los porcentajes de germinación fueron muy bajos: el porcentaje más alto que se obtuvo fue de $4.7 \%$ y en muchos de los lotes no germinó ninguna semilla (cuadro 1).

Se encontraron diferencias altamente significativas entre la germinación de las semillas protegidas de la depredación y las expuestas a este factor $(F=672.85$; g.l. $=$ $1 ; P<0.00001)$. Bajo todas las especies probadas y en los claros, la depredación fue muy alta y en algunos de los lotes se perdieron todas las semillas. Los principales depredadores observados fueron las hormigas.

Por el contrario, no se encontraron diferencias significativas para el factor asociación, es decir, no hubo diferencias entre la germinación en los claros y bajo la copa de las nodrizas $(F=1.041$; g.l. $=4 ; P=0.3877)$. En cuanto a la orientación de los lotes hacia los diferentes puntos cardi-

Cuadro 1. Valores promedio ( \pm 1 D.E.) del porcentaje de germinación de semillas de Pterocereus gaumeri obtenido en los diferentes tratamientos aplicados.

\begin{tabular}{|c|c|c|c|c|c|c|}
\hline Protegidas de la depredación & Orientación & $\begin{array}{l}\text { Amyris } \\
\text { atenuata }\end{array}$ & $\begin{array}{l}\text { Guaiacum } \\
\text { sanctum }\end{array}$ & $\begin{array}{l}\text { Acacia } \\
\text { gaumeri }\end{array}$ & $\begin{array}{l}\text { Malpighia } \\
\text { punicifolia }\end{array}$ & Claros \\
\hline & $\mathrm{N}$ & $30 \pm 14.72$ & $35.3 \pm 12.4$ & $42 \pm 6.5$ & $30.7 \pm 20.4$ & $35.3 \pm 19.9$ \\
\hline & $S$ & $32 \pm 16.4$ & $35.3 \pm 8.3$ & $40 \pm 7.4$ & $26.7 \pm 14.3$ & $44.7 \pm 21.6$ \\
\hline \multirow[t]{4}{*}{ Sí } & $\mathrm{E}$ & $29.3 \pm 11.8$ & $30 \pm 3.3$ & $18.7 \pm 6.9$ & $36.7 \pm 6.2$ & $32 \pm 21.6$ \\
\hline & $\mathrm{O}$ & $33.3 \pm 15$ & $42 \pm 16$ & $36.7 \pm 14.3$ & $24 \pm 18.3$ & $43.3 \pm 21.9$ \\
\hline & $\mathrm{N}$ & $1.3 \pm 1.8$ & $2.7 \pm 5.9$ & 0 & 0 & $2.7 \pm 5.9$ \\
\hline & S & $2 \pm 2.9$ & $0.7 \pm 1.4$ & 0 & $1.3 \pm 1.8$ & $0.7 \pm 1.4$ \\
\hline \multirow[t]{2}{*}{ No } & $\mathrm{E}$ & 0 & $2 \pm 2.9$ & $0.6 \pm 1.4$ & $4.6 \pm 6.9$ & $4.7 \pm 7.3$ \\
\hline & $\mathrm{O}$ & $4 \pm 7.2$ & $2.7 \pm 5.9$ & 0 & 0 & $4 \pm 8.9$ \\
\hline
\end{tabular}


nales, este factor no mostró un efecto significativo en la germinación $(F=0.113 ; \mathrm{g} .1=3 ; P=0.9527)$. Con respecto a las interacciones entre factores, no se encontraron diferencias significativas entre dichas interacciones, ni en la interacción triple $(P>0.1651$ para cada una de las interacciones). Cabe señalar que la germinación obtenida para las semillas probadas bajo condiciones controladas en el laboratorio fue de $97.3 \%$.

Supervivencia. Se encontraron diferencias significativas en las curvas de supervivencia de las plántulas que crecieron bajo cada especie de nodriza y las que se encontraban expuestas a la radiación, creciendo en los claros (LR = 67.098; g.l. $=4 ; P<0.05)$. Durante los meses de octubre a enero la mortalidad de las plántulas que crecieron en los claros fue similar a la de las que se encontraban debajo de las nodrizas, pero a partir del mes de marzo, la mortalidad de las plántulas en los claros se incrementó significativamente (figura 1).

Durante el período de marzo a junio, 90\% de las plántulas que se encontraban en los claros murieron, lo cual coincide con la época más fuerte del periodo de sequía (febrero a mayo). Durante el primer año de estudio, en la época de sequía se registró sólo $12 \%$ de la precipitación total, siendo notable que durante el mes de febrero no hubo precipitación. Asimismo, la temperatura máxima $\left(43.5^{\circ} \mathrm{C}\right)$ se presentó en mayo.

Se encontraron diferencias significativas entre la supervivencia de plántulas bajo Amyris atenuata y los claros (LR = 61.644; g.l. $=1 ; P<0.05)$; entre la registrada bajo Malpighia punicifolia y los claros (LR = 73.090; g.l. $=1 ; P$ $<0.05$ ); entre la obtenida bajo Acacia gaumeri y los claros (LR = 98.284; g.l. $=1 ; P<0.05)$; y entre la evaluada bajo Guaiacum sanctum y los claros $(\mathrm{LR}=44.436$; g.l. $=1 ; P$ $<0.05)$.

No se encontró una mortalidad diferencial entre las plántulas que estaban creciendo bajo las cuatro especies seleccionadas como nodrizas $(\mathrm{LR}=6.51$; g.l. $=3 ; P<0.05$; figura 1), ni tampoco se observó ningún efecto significativo de la orientación de las plántulas (norte, sur, este y oeste) sobre su supervivencia $(\mathrm{LR}=2.82$; g.l. $=1 ; P<0.05$; figura 2).

En contraste, la depredación mostró tener un efecto importante en la mortalidad de plántulas. Al término del primer año de estudio los porcentajes de supervivencia registrados para las plántulas protegidas de la depredación (34\%) fueron significativamente mayores que los de las expuestas a los depredadores $(12 \%) ;(\mathrm{LR}=11.90$; g.1. $=1$; $P<0.05$; figura 3).

Por otra parte, el análisis de varianza permite concluir que existen diferencias significativas en el factor protección $(F=287.003$; g.l. $=1 ; P<0.000001)$ y en el factor asociación $(F=14.3676 ;$ g.l. $=4 ; P<0.000001)$. Con respecto a la interacción entre factores, sólo se encontraron diferencias significativas en la interacción protección-asociación $(F=12.7338$; g.l. $=4 ; P<0.000001)$. No hubo diferencias significativas en el factor orientación $(F=$ 0.3173 ; g.l. $=3 ; P=0.8129)$, ni en las otras interacciones posibles entre factores $(P>0.3765)$.

\section{Discusión}

A diferencia de lo que se ha reportado para otras especies de cactáceas columnares (Turner et al., 1966; Nobel, 1980; Franco y Nobel, 1989; Valiente-Banuet y Ezcurra, 1991; Esparza-Olguín et al., 2002), en nuestros experimentos no se registró una mortalidad diferencial de semillas asociada a los diferentes microambientes. Es decir, el efecto de los claros y sus factores asociados (radiación, temperatura y evaporación) no fueron determinantes para generar diferencias significativas entre la germinación que ocurre bajo la copa de las plantas y en los claros, y tampoco entre especies. Esto sugiere que para la germinación de $P$. gaumeri no es indispensable que las semillas se encuentren al abrigo de las plantas nodriza, probablemente debido a que durante el período de lluvias, época en la que germinan las semillas de esta especie, las condiciones ambientales no son tan drásticas como ocurre durante la época de sequía.

A pesar de que no se observaron diferencias significativas, es interesante notar que en el caso de los claros se presentó una mayor germinación en los lotes colocados en la parte sur (respecto al centro) de los mismos. Esto probablemente se deba al efecto de los árboles ubicados en el borde sur del claro, cuya sombra se proyecta hacia el norte la mayor parte del año, haciendo que la zona sur del claro sea más sombreada y húmeda que el área norte del mismo. Esto pudiera generar en los claros un efecto inverso a la acción de las nodrizas, las cuales brindan condiciones más favorables en dirección al norte.

Por otra parte, los resultados de este estudio mostraron que las plantas nodriza seleccionadas no brindaron protección alguna a las semillas de $P$. gaumeri contra la depredación. No se presentó una depredación diferencial de semillas entre claros y bajo las plantas nodriza, aun cuando entre las especies seleccionadas se encontraba Malpighia punicifolia, que es un arbusto con hábito semirastrero.

Con relación a la supervivencia de las plántulas, los resultados encontrados son completamente diferentes. La mortalidad de las plántulas ubicadas debajo de las plantas nodriza fue significativamente menor que las ubicadas en los claros; al cabo de un año todos los individuos establecidos en los claros se murieron, lo cual coincide con lo reportado para otras especies de cactáceas columnares como Carnegia gigantea (Niering et al., 1963; Turner et al., 1966; Steenbergh y Lowe, 1977, 1983; Nobel, 1980; Vandermeer, 1980; Hutto et al., 1986; Franco y Nobel, 1989), Cephalocereus hoppenstedtii (Valiente-Banuet et 


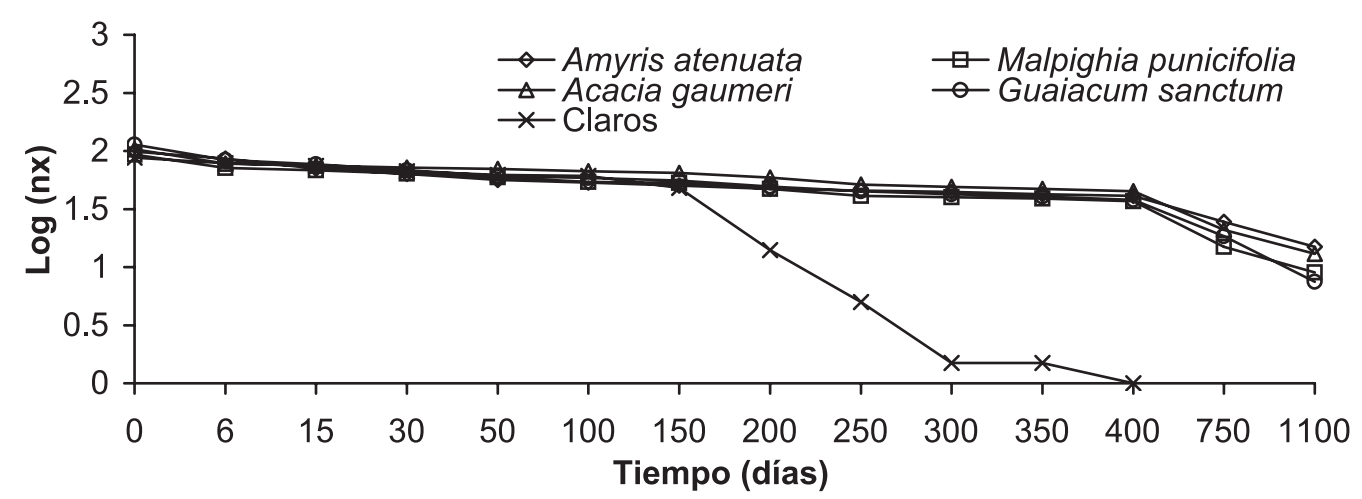

Figura 1. Curvas de supervivencia de las plantas que crecieron bajo diferentes especies de plantas nodriza y las que crecieron en los claros durante un período de 1,100 días $(\mathrm{LR}=67.088$; g.1. = 1; $P<0.05)$.

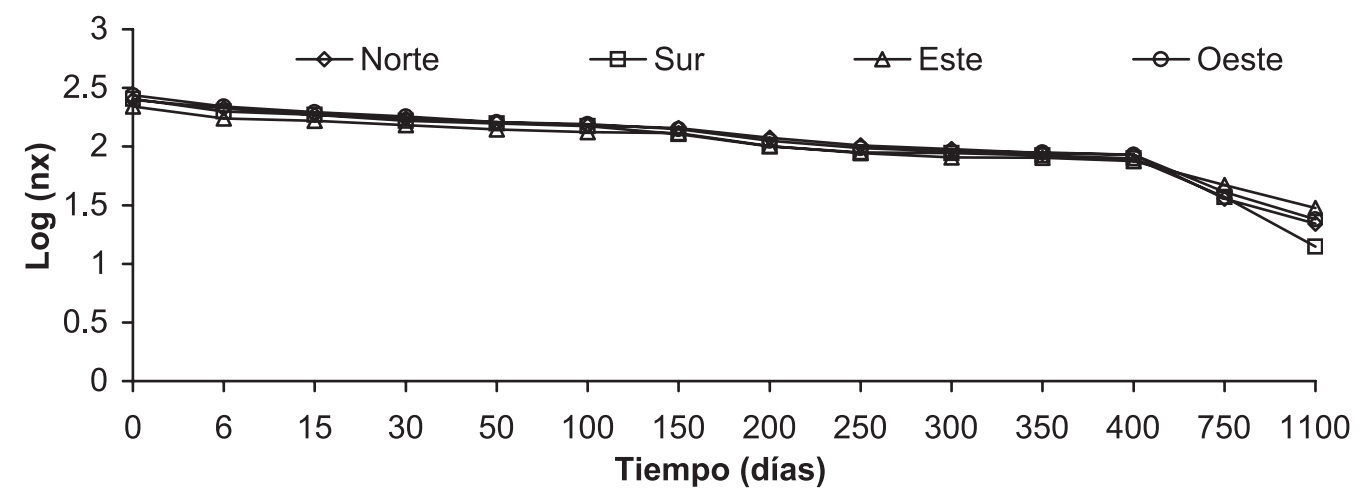

Figura 2. Curvas de supervivencia de las plántulas que crecieron en los diferentes puntos cardinales. Se incluyeron todos los tratamientos (plantas nodriza, claros, expuestas y protegidas de la depredación).

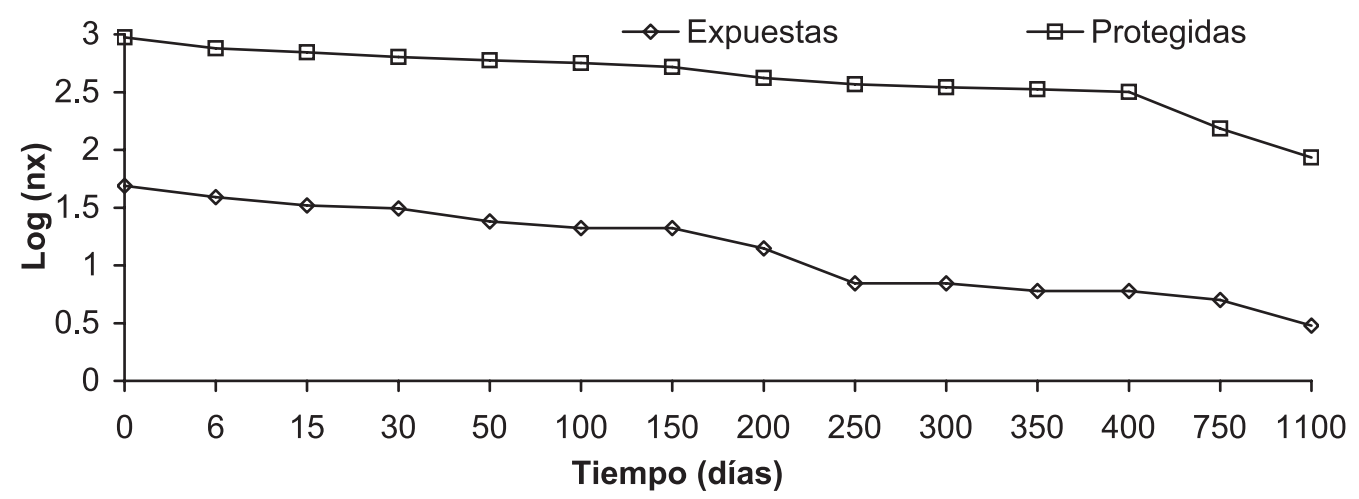

Figura 3. Curvas de supervivencia de las plántulas protegidas y expuestas a los depredadores durante un período de estudio de 1,100 días. 
al., 1991a), Neobuxbaumia tetetzo (Valiente-Banuet y Ezcurra, 1991), Stenocereus thurberii (Arriaga et al., 1993) y Neobuxbaumia macrocephala (Esparza-Olguín et al., 2002).

Los datos de mortalidad registrados durante los meses de mayor sequía sugieren que la radiación y sus factores asociados (temperatura y evaporación), además de la escasa precipitación, crearon condiciones microambientales muy diferentes entre los claros y debajo de la copa de los árboles y arbustos, las cuales se reflejan en la mortalidad diferencial de las plántulas que crecieron en cada condición. Sin embargo, no se encontraron diferencias significativas en la supervivencia de las plántulas que crecieron bajo las diferentes especies de nodriza, lo cual sugiere que las condiciones microclimáticas no fueron diferentes entre especies, aun cuando dos de las especies estudiadas son caducifolias. En este sentido, cabe señalar que muchas de las especies caducifolias de las selvas bajas tienen la capacidad de producir hojas como respuesta a eventos de precipitación que se presentan durante el período de sequía. Durante el primer período de sequía, posterior al establecimiento del experimento, en marzo de 1999 se registraron $48 \mathrm{~mm}$ de precipitación, con lo cual las hojas pudieron rebrotar, amortiguando las condiciones microclimáticas debajo de la copa de las especies caducifolias; además, las hojas que caen al piso de la selva contribuyen a la protección de las plántulas. Aunado a esto, la mayor complejidad estructural de la selva baja caducifolia con relación a la vegetación de las zonas desérticas podría explicar porqué no se encontraron diferencias significativas en la supervivencia de las plántulas establecidas bajo las diferentes especies seleccionadas como nodrizas.

Aunque se ha reportado que el fenómeno de nodricismo no tiene para algunas especies de cactáceas la misma importancia en las selvas bajas que en las zonas desérticas (Arriaga et al., 1993), en el caso de P. gaumeri la supervivencia de plántulas fue mayor bajo las plantas nodriza que en los claros. Cabe señalar que la selva baja con cactáceas candelabriformes del norte de Yucatán no es una selva con dosel continuo, sino que presenta numerosos espacios abiertos producto del afloramiento de roca caliza (lajas) y la gran pedregosidad de sus suelos. En este ambiente muy heterogéneo las nodrizas proporcionan a las plántulas de esta especie microambientes con menos radiación solar directa que en los sitios abiertos, y consecuentemente, con temperaturas más bajas y menor demanda evaporativa, lo cual las protege de un incremento en la temperatura de sus tejidos y de la pérdida de agua por transpiración (Gibson y Nobel, 1986). Un elemento adicional es el hecho de que los claros generalmente se presentan en sitios con afloramientos de roca y suelos muy someros, lo que limita su capacidad de retención de humedad, lo que contribuye a explicar la alta mortalidad de plántulas en estos sitios.

Se ha reportado que para algunas cactáceas expuestas a la radiación solar directa, su temperatura puede incrementarse $15^{\circ} \mathrm{C}$ o más por encima de la temperatura del aire. Para las plántulas de algunas cactáceas esto podría representar una condición muy peligrosa, ya que no tienen tanta capacidad de acumular calor en el tallo, como lo hacen los individuos adultos (Gibson y Nobel, 1986). En este sentido, es importante avanzar en el conocimiento de los mecanismos fisiológicos que permiten la termorregulación de los individuos de las especies sometidas a condiciones de radiación extrema en esta fase de su ciclo de vida.

Otro aspecto interesante a explorar es la disyuntiva de las plántulas entre crecer y sobrevivir, ya que si bien la supervivencia de las cactáceas bajo la sombra de otras especies puede ser mayor, su crecimiento se puede ver sustancialmente limitado. Franco y Nobel (1989) han sugerido que el déficit de luz (RFA) experimentado por cactáceas bajo sombra puede ser compensado por niveles altos de fertilidad encontrados en estos microambientes, aunque esta hipótesis no ha sido probada en todos los ambientes donde se ha estudiado (e.g. Valiente-Banuet y Ezcurra, 1991). Por otra parte, se ha reportado que las plántulas de algunas especies de cactáceas modifican su metabolismo fotosintético bajo condiciones adecuadas de agua (Szarek y Ting, 1975). Sería importante explorar si este mecanismo se presenta en las plántulas de $P$. gaumeri.

En conclusión, a diferencia de los resultados que se han encontrado para otras especies de cactáceas columnares, las semillas de $P$. gaumeri no requieren de plantas nodriza para su germinación. Sin embargo, la protección que brindan las plantas de la selva fue fundamental para la supervivencia de las plántulas de esta especie.

Para algunas especies de cactáceas, la depredación de semillas constituye un fenómeno importante en la dinámica de sus poblaciones, ya sea que éstas sean removidas del fruto cuando aún se encuentran en la planta madre o bien cuando ya cayeron al suelo (Steenbergh y Lowe, 1969, 1977; Valiente-Banuet y Ezcurra, 1991). En el caso particular de $P$. gaumeri se ha observado que alrededor de $85 \%$ de los frutos son depredados cuando aún están verdes (Méndez et al., 2005), lo que contribuye a que, contrario a lo encontrado para otras especies de cactáceas, como Neobuxbaumia tetetzo (Valiente-Banuet y Ezcurra, 1991), las semillas de P. gaumeri no se encuentran de manera abundante en el piso de la selva, y más aún, las que llegan al suelo están sujetas a una intensa depredación. Los resultados obtenidos mostraron que en los experimentos de las semillas expuestas a la depredación solamente germinó en promedio $1.7 \%$, proporción menor a la encontrada para $N$. tetetzo en el Valle de Zapotitlán, Puebla (Valiente-Banuet y Ezcurra, 1991). Estos resultados nos permiten sostener que la depredación de las semillas y la alta mortalidad de plántulas juegan un papel fundamental en el ciclo de vida de esta especie, limitando el reclutamiento de nuevos individuos a las poblaciones de $P$. gaumeri. 
La suma de factores que afectan el proceso reproductivo de $P$. gaumeri, desde la aborción de un elevado número de botones y flores, la alta depredación de frutos inmaduros (Méndez et al., 2005), la gran depredación de semillas en el suelo y la alta mortalidad de plántulas expuestas a condiciones ambientales extremas, contribuye a explicar la rareza de esta especie.

De las cactáceas columnares presentes en la selva baja de Yucatán (Pilosocereus gaumeri, Stenocereus eichlamii y Pachycereus sp.), Pterocereus gaumeri es la especie que tiene la distribución más restringida y la de menor densidad poblacional. Aunado a esto, el limitado reclutamiento de nuevos individuos a las poblaciones y el lento crecimiento de sus individuos la hacen ser una especie muy vulnerable a las perturbaciones de su hábitat, por lo que es urgente tomar medidas para su conservación.

\section{Agradecimientos}

Agradecemos a Paulino Simá y Luis Simá su valiosa ayuda en el trabajo de campo. Asimismo, agradecemos a Jorge A. Meave y a dos revisores anónimos por su revisión crítica, útiles comentarios y sugerencias que mejoraron notablemente el documento final. El presente trabajo contó con el apoyo financiero del CONACYT (beca doctoral No. 93624) y del Sistema Regional de Investigación Justo Sierra (proyecto No. 950603).

\section{Literatura citada}

Arriaga L., Maya Y., Díaz S. y Cancino J. 1993. Association between cacti and nurse perennials in a heterogeneous tropical dry forest in northwestern Mexico. Journal of Vegetation Science 4:349-356.

Bravo-Hollis H. 1978. Las Cactáceas de México. Vol. 1. Universidad Nacional Autónoma de México, México, D.F.

Contreras C. y Valverde T. 2002. Evaluation of the conservation status of a rare cactus (Mammillaria crucigera) through the analysis of its population dynamics. Journal of Arid Environments 51:89-102.

Espadas C., Durán R. y Argáez J. 2003. Phytogeographic analysis of taxa endemic to the Yucatan Peninsula using geographic information systems, the domain heuristic method and parsimony analysis of endemicity. Diversity and Distributions 9:313-330.

Esparza-Olguín I., Valverde T. y Vilchis-Anaya I. 2002. Demographic analysis of a rare columnar cactus (Neobuxbaumia macrocephala) in the Tehuacán Valley, México. Biological Conservation 103:349-359.

Fielder P. y Jain S. 1992. Conservation Biology: The Theory and Practice of Nature Conservation, Preservation and Management. Chapman and Hall, Nueva York.

Fowler N. 1986. The role of competition in plant communities in arid and semiarid regions. Annual Review of Ecology and Systematics 17:89-110.

Franco A. y Nobel P. 1989. Effect of nurse plants on the microhabitat and growth of cacti. Journal of Ecology 77:870-886.
García-Moya E. y Mckeel M. 1970. Contribution of shrubs in the economy of a desert-wash plant community. Ecology 51:8188.

Gibson A. y Nobel P. 1986. The Cactus Primer. Harvard University Press, Cambridge, Massachusetts.

Godínez-Álvarez H. y Valiente-Banuet A. 1998. Germination and early seedling growth of Tehuacan Valley cacti species: the role of soils and seed ingestion by dispersers on seedling growth. Journal of Arid Environments 39:21-31.

Godínez-Álvarez H., Valiente-Banuet A. y Valiente-Banuet B.L. 1999. Biotic interactions and the population dynamics of the long-lived columnar cactus Neobuxbaumia tetetzo in the Tehuacán Valley, Mexico. Canadian Journal of Botany 77:203208.

Godínez-Álvarez H., Valverde T. y Ortega-Baes P. 2003. Demographic trends in the Cactaceae. The Botanical Review 69:173-203.

Harrison S. 1993. Species diversity, spatial scale, and global change. En: Kareiva P., Kingsolver J. y Huey R. Eds. Biotic Interactions and Global Change, pp. 388-401, Sinauer, Sunderland.

Hutto R., McAuliffe J. y Hogan L. 1986. Distributional associates of the saguaro (Carnegiea gigantea). Southwestern Naturalist 31:469-476.

Mandujano M., Méndez I. y Golubov J. 1998. The relative contributions of sexual reproduction and clonal propagation in Opuntia rastrera from two habitats in the Chihuahuan Desert. Journal of Ecology 86:911-921.

McAuliffe R. 1984. Sahuaro-nurse tree associations in the Sonoran desert: competitive effects on Sahuaro. Oecologia 64:319-321.

McAuliffe R. 1988. Markovian dynamics of simple and complex desert plants communities. American Naturalis 131:459-490.

Méndez M., Durán R., Dorantes A., Dzib G., Simá L., Simá P. y Orellana R. 2005. Floral demography and reproductive system of Pterocereus gaumeri, a rare columnar cactus endemic to Mexico. Journal of Arid Environments 62:363-376.

Méndez M., Durán R., Olmsted I. y Oyama K. 2004. Population dynamics of Pterocereus gaumeri, a rare and endemic columnar cactus of Mexico. Biotropica 36:492-504.

Miranda F. y Hernández-X. E. 1963. Los tipos de vegetación de México y su clasificación. Boletín de la Sociedad Botánica de México 28:29-179.

Niering W., Wittaker R. y Lowe C. 1963. The saguaro: a population in relation to environment. Science 142:15- 23.

Nobel P. 1980. Morphology nurse plants and minimum apical temperatures for young Carnegiea gigantea. Botanical Gazette 141:188-191.

Nolasco H., Vega-Villasante F. y Diaz-Rondero A. 1997. Seed germination of Stenocereus thurberi (Cactaceae) under different solar irradiation levels. Journal of Arid Environments 36:123-132.

Olmsted I., Durán R., González-Iturbe J.A., GranadosCastellanos J. y Tun-Dzul F. 1999. Vegetación de la Península de Yucatán. En: Chico-Ponce de León P. y García A. Eds. Atlas de los Procesos Territoriales de Yucatán, pp. 183-194, Universidad Autónoma de Yucatán, Mérida.

Ollin G., Alcorn S.M. y Alcorn J.M. 1989. Dispersal of viable saguaro seeds by white-winged doves (Zenaida asiatica). Southwestern Naturalist 34:282-284. 


\section{Germinación y ESTABlecimiento De PTEROCEREUS GAUMERI}

Pyke D.A. y Thompson J.N. 1986. Statistical analysis of survival and removal rate experiments. Ecology 67:240-245.

Reveal J.L. 1981. The concepts of rarity and population threats in plant communities. En: Morse L.E. y Henefin M.S. Eds. Rare Plant Conservation, pp. 41-47, The New York Botanical Garden, Nueva York.

Rodríguez-Ortega C.E. y Ezcurra E. 2000. Distribución espacial en el hábitat de Mammillaria pectinifera y M. carnea en el valle de Zapotitlán Salinas, Puebla, México. Cactáceas y Suculentas Mexicanas 45:4-14.

Ruedas M., Valverde T. y Castillo S. 2000. Respuesta germinativa y crecimiento de plántulas de Mammillaria magnimamma (Cactaceae) bajo diferentes condiciones ambientales. Boletín de la Sociedad Botánica de México 66:25-35.

Shreve F. 1931. Physical conditions in sun and shade. Ecology 12:96-104.

Steenbergh W.F. y Lowe C.H. 1969. Critical factors during the first years of life of the saguaro (Cereus giganteus) at the Saguaro National Monument, Arizona. Ecology 50:825-834.

Steenbergh W.F. y Lowe C.H. 1977. Ecology of the Saguaro: II. Reproduction, Germination, Establishment, Growth and Survival of the Young Plants. National Park Service Scientific Monograph Series No. 8, U.S. Government Printing Office, Washington, D.C.

Steenbergh W.F. y Lowe C.H. 1983. Ecology of the Saguaro: III. Growth and Demography. National Park Service Scientific Monograph Series No. 178, U.S. Government Printing Office, Washington, D.C.

Suzán H., Nabhan G.P. y Patten D.T. 1996. The importance of Olneya tesota as a nurse plant in the Sonoran Desert. Journal of Vegetation Science 7:635-644.
Szarek R.S. y Ting P.I. 1975. Physiological responses to rainfall in Opuntia basilaris (Cactaceae). American Journal of Botany 62:602-609.

Turner R., Alcorn S., Olin G. y Booth J. 1966. The influence of shade, soil and water on saguaro seedling establishment. Botanical Gazette 127:95-102.

Valiente-Banuet A., Bolongaro-Crevenna A., Briones O., Ezcurra E., Rosas M., Núñez M., Barnard G. y Vázquez E. 1991a. Spatial relationships between cacti and nurse shrubs in a semiarid environment in central Mexico. Journal of Vegetation Science 2:15-20.

Valiente-Banuet A. y Ezcurra E. 1991. Shade as a cause of the association between the cactus Neobuxbaumia tetetzo and the nurse plant Mimosa luisiana in the Tehuacán Valley, Mexico. Journal of Ecology 79:961-971.

Valiente-Banuet A., Vite F. y Zavala-Hurtado A. 1991b. Interaction between the cactus Neobuxbaumia tetetzo and the nurse shrub Mimosa luisana. Journal of Vegetation Science 2:11-14

Vandermeer J. 1980. Saguaros and nurse trees: a new hypothesis to account for population fluctuations. Southwestern Naturalist 25:357-360.

Walter K.S. y Gillett H.J. 1998. 1997 IUCN Red List of Threatened Plants. Compiled by the World Conservation Monitoring Center. IUCN-The World Conservation Union, Gland, Suiza.

Zar J.H. 1984. Biostatistical Analysis. 2a ed. Printice-Hall, Englewood Cliffs, Nueva Jersey.

Zúñiga B., Malda G. y Suzán H. 2005. Interacciones plantanodriza en Lophophora diffusa (Cactácea) en un desierto subtropical de México. Biotropica 37:351-356.

Fecha de recepción: 12 de enero de 2006

Versión corregida: 25 de octubre de 2006

Aceptado: 25 de octubre de 2006 